\title{
A comparison between 2D and 3D planning of high-dose-rate vaginal cuff brachytherapy in patients with stage $\mathrm{I}-\mathrm{Il}$ endometrial cancer using cobalt-60
}

\author{
Farnaz Amouzegar Hashemi, MD!, Sepideh Mansouri, MD, MPH',2, Mahdi Aghili, MD!, Ebrahim Esmati, MD!, \\ Mohammad Babaei, MD!, Arefeh Saeedian, MDl,3, Sepand Moalej, Ramin Jaberi, PhD ${ }^{5}$ \\ IRadiation Oncology Research Center (RORC), Cancer Institute, Tehran University of Medical Sciences, Tehran, Iran, ${ }^{2}$ Recombinant Proteins \\ Department, Breast Cancer Research Center, Motamed Cancer Institute, ACECR, Tehran, Iran, ${ }^{3}$ Department of Radiation Oncology, \\ Imam Khomeini Hospital Complex, Cancer Institute, Tehran University of Medical Sciences, Tehran, Iran, ${ }^{4}$ Western University of Health Sciences \\ Pomona, USA, ${ }^{5}$ Physics Department of Radiation Oncology, Tehran University of Medical Sciences, Tehran, Iran
}

\begin{abstract}
Purpose: Post-surgery radiation can reduce the risk of loco-regional relapse in high-intermediate-risk endometrial cancer. High-dose-rate vaginal cuff brachytherapy (HDR-BRT) is an acceptable method of radiation in majority of endometrial cancer cases. Although 2D planning is frequently used for treatment based on physical examination without any imaging, measurement of the dose received by organs-at-risk (OARs) is not possible. Therefore, the present study was the first to compare dose delivered to target and OARs in 2D vs. 3D planning in patients treated with cobalt-60 source.

Material and methods: In this study, organs including vagina wall, bladder, rectum, and sigmoid were contoured on computed tomography (CT) scan images of 37 endometrial cancer patients, and doses delivered to organs were recorded. Statistics, such as $\mathrm{D}_{90}, \mathrm{D}_{99}, \mathrm{~V}_{100}, \mathrm{~V}_{150}, \mathrm{~V}_{200}, \mathrm{D}_{0.1 \mathrm{cc}}, \mathrm{D}_{1 \mathrm{cc}}$ and $\mathrm{D}_{2 \mathrm{cc}}$ were determined.

Results: $\mathrm{D}_{90}$ and $\mathrm{D}_{99}$ were lower in $3 \mathrm{D}$ treatment planning in comparison with $2 \mathrm{D}$. Although $\mathrm{V}_{100}$ was more in $3 \mathrm{D}$ planning, $\mathrm{V}_{150}$ and $\mathrm{V}_{200}$ were less. Analyzing $\mathrm{D}_{0.1 \mathrm{cc}}, \mathrm{D}_{1 \mathrm{cc}}$ and $\mathrm{D}_{2 \mathrm{cc}}$ of OARs revealed that doses given to rectum, sigmoid, and bladder were less in 3D planning compared to $2 \mathrm{D}$.

Conclusions: Comparison of 2D and 3D planning results showed that 3D planning could deliver an appropriate dose to the target while sparing more OARs.

J Contemp Brachytherapy 2021; 13, 5: 526-532 DOI: https://doi.org/10.5114/jcb.2021.110312
\end{abstract}

Key words: dosimetry, high-dose-rate brachytherapy, 2D and 3D planning, endometrial cancer, organs-at-risk.

\section{Purpose}

Endometrial cancer is one of the most prevalent gynecological cancer, with an incidence rate of more than 380,000 patients per year, and is responsible for approx. 90,000 deaths per year worldwide [1]. The median age of patients is around 60-65 years, and majority of patients are diagnosed at an early stage [2]. Early-stage patients, who underwent total abdominal hysterectomy (TAH) and bilateral salpingo-oophorectomy (BSO) have a fiveyear overall survival (OS) of around 95\% [3]. In addition, patients may need to receive adjuvant therapies according to certain indications, including high-grade, deep stromal, myometrial, and lymphovascular invasion, lymph node(s) metastasis, and margin involvement [4-6]

According to PORTEC-1 (post-operative radiation therapy in endometrial cancer) trial [4, 7] and GOG 99
(Gynecological Oncology Group) trial 99 [5], external beam radiation (EBRT) demonstrated utility in high-intermediate-risk patients (stage IB grade 1 , any patients with grade 2 , and stage IA grade 3 were considered as high-intermediate-risk) in terms of reducing local failure; however, OS was not shown to improve. PORTEC-2 trial [8] with high-intermediate-risk patients without staging pelvic lymphadenectomy (LND), showed no difference between high-dose-rate brachytherapy (HDR-BRT) and EBRT in vaginal cuff recurrences. However, EBRT was demonstrated to be superior in pelvic control in comparison with HDR-BRT alone. Ten years follow-up of PORTEC-2 trial confirmed that vaginal cuff BRT could be the standard of treatment for high-intermediate-risk early-stage endometrial cancer [9]. Moreover, in advanced cases, additional use of EBRT in HDR-BRT is recommended because of reduced pelvic recurrences in EBRT and BRT [10]. 
As shown in PORTEC-2, vaginal cuff brachytherapy in endometrial cancer patients had excellent therapeutic outcomes [8]. Application of a single-channel vaginal cylinder to cover a $5 \mathrm{~mm}$ depth of vaginal mucosa is a simple and effective technique. Therefore, it became the most popular instrument to perform HDR-BRT [11]. Worldwide, the most common dosimetry method in patients who had undergone HDR-BRT is two-dimensional (2D) technique. According to physical examination, length and diameter of appropriate vaginal cylinder should be considered [12]. Since there is no cross-sectional anatomy imaging, dosimetry of target volume and OARs are impossible. In contrast, in image-based three-dimensional (3D) planning, a treatment is designed according to contours delineated and dose constraints. There are few studies comparing 2D vs. 3D planning in HDR-BRT of endometrial cancer. Kim et al. [13] compared 2D and 3D planning in endometrial cancer treated with HDR-BRT. They planned 2D plans through manual optimization points and also planned 3D in CT images. They revealed that all determinants, such as $\mathrm{D}_{90}, \mathrm{D}_{100}, \mathrm{~V}_{100}, \mathrm{~V}_{150}$, and $\mathrm{V}_{200}$ were lower in $3 \mathrm{D}$ comparing to $2 \mathrm{D}$. OARs, including rectum and bladder received lower doses in $3 \mathrm{D}$ compared to 2D. Therefore, this study showed that 3D has superiority over 2D in terms of saving OARs without compromising target volume dose. A study by Humphrey et al. [14] demonstrated that CT scan can help in cylinder reposition to reduce air gap in roughly $7 \%$ of patients. Another study by Russo et al. [15] focusing on OARs (bladder and rectum) showed that dose calculation in 2D plans according to ICRU (International Commission on Radiation Units and Measurements) points could not accurately estimate delivered dose. It was shown that maximum dose and $\mathrm{D}_{2 \mathrm{cc}}$ calculated in 3D plans were more accurate than ICRU dose, and consequently, the authors concluded that ICRU could not represent dose delivered to OARs. Recently, Gultekin et al. [16] reported that 3D planning in HDR-BRT of endometrial cancer patients could spare OARs more than frequently used methods, which deliver prescribed dose to a $5 \mathrm{~mm}$ distance from the surface of cylinder or at its' surface.

The most popular source applied in gynecologic brachytherapy was iridium-192 ( $\left.{ }^{192} \mathrm{Ir}\right)$, due to its small size and suitable physical aspects, until miniaturized cobalt $-60\left({ }^{60} \mathrm{Co}\right)$ was introduced into clinical practice. Here, the advantages and disadvantages of ${ }^{60} \mathrm{Co}$ administration will be compared to ${ }^{192} \mathrm{Ir}$ [17]. First, a longer half-life of ${ }^{60} \mathrm{Co}$ (5.3 years) in comparison to ${ }^{192} \operatorname{Ir}(74$ days) resulted in reduced operating costs, with logistical and economic advantages [18-21]. For example, in a definite interval time, 25 source replacements of ${ }^{192} \mathrm{Ir}$ would be needed in comparison to just a single application of ${ }^{60} \mathrm{Co}$ [17]. Second, despite the fact that these two sources could have different physical characteristics [17], studies showed that dose distribution is identical. Also, toxicity and clinical outcomes of ${ }^{192} \mathrm{Ir}$ and ${ }^{60} \mathrm{Co}$ were similar. However, there is a limitation regarding administration of ${ }^{60} \mathrm{Co}$, which emits high-energy gamma rays, and that requires the staff to provide a thicker shielding material [17].
There are few studies that compared 2D and 3D planning with different methods of dose calculation in 2D plans, including ICRU point [15], manual placing of optimization point [13], dose delivery to a $5 \mathrm{~mm}$ depth of vagina wall, or dose delivery to the surface of cylinder [16]. In the current study, a $5 \mathrm{~mm}$ depth of the vagina was chosen as dose delivering point in 2D planning, because this depth is comparable with vagina thickness contoured in 3D planning, and at the same time, the bias of comparing PTV coverage between 2D and 3D plans was limited. To the authors' knowledge, the present study is the first research to compare $2 \mathrm{D}$ with $3 \mathrm{D}$ planning through administration of ${ }^{60} \mathrm{Co}$. The aim of this study was to determine whether 3D plans provided better target coverage, and to explore differences in dose delivered to OARs, when considering cross-sectional imaging and contours in $3 \mathrm{D}$ planning compared to $2 \mathrm{D}$ controls using ${ }^{60} \mathrm{Co}$ as a brachytherapy source.

\section{Material and methods \\ Population}

This cross-sectional study was conducted in radiation oncology departments of two different hospitals. Healthcare professionals and treatment protocols of both hospitals were the same, as both of them belong to one university. According to Kim et al. [13] who considered dose delivered to $1 \mathrm{cc}$ of rectum in 2D plan (88\%) and 3D plan (93\%), acceptable difference was considered as $15 \%$, first error was less than $5 \%$, and power ( 1 - beta) was $90 \%$, with thirty-five patients analyzed through non-inferiority sample size formula [22]. Forty-one confirmed cases of early-stage endometrial cancer of IA to IIB FIGO stages were enrolled, and underwent total abdominal hysterectomy and bilateral salpingo-oophorectomy (TAH-BSO) and subsequent radiotherapy using HDR-BRT as monotherapy. All the patients were provided with written informed consents, and their data were coded and kept confidential. Furthermore, study protocol was approved by an ethics committee of affiliated university.

\section{CT scanning and contouring}

The patients underwent a non-contrast pelvic CT scan (Sommatom DR ${ }^{\circledR}$, Siemens, Germany), with slices thickness of 3 to 5 millimeters from mid-pubis to the lower border of ischial tuberosity. Patients with sub-total hysterectomy or those with cervical tissue remnants in CT images were excluded from the study after scanning. Vagina was considered as the target volume. Rectum, sigmoid, and bladder were delineated as OARs. Vaginal length for clinical target volume (CTV) was defined according to grade and histology of the tumor. CTV was contoured superiorly $2.5 \mathrm{~cm}$ upper cylinder, and could be modulated with vagina in CT images [13]. Patients with grade 1 and 2 endometrioid carcinoma were treated with a $4 \mathrm{~cm}$ length cylinder, if $\leq 50 \%$ of myometrial invasion and a $5 \mathrm{~cm}$ length cylinder in case of $>50 \%$ myometrial involvement. Six centimeters of the vagina were treated in grade 3 endometrioid carcinoma, clear cell carcinoma, and serous papillary carcinoma [12]. In addition, thick- 
ness of target volume was contoured based on CT scan slices (vaginal width was considered around $5 \mathrm{~mm}$ and lessened based on adjacent OARs, or increased according to thickness seen on CT slices), and length of the vagina was modulated based on clinico-pathological aspects.

The optimum plan was described when the target volume received more significant percentage of the prescribed dose, while dose delivered to OARs was as little as possible. The upper and lower borders of the rectum were set at S2-S3 interspace and puborectalis muscles, respectively. In addition, the sigmoid was contoured from true pelvis to recto-sigmoid junction, and the lower border of bladder was also considered as levator ani muscle. Contours were outlined by a physician and then confirmed by another physician and a physics master.

\section{D vs. 3D planning}

After these pre-requisites, the treatment was planned using HDR Plus v. 8.2.3 ${ }^{\circledR}$ software and administration of TG-43 algorithm, according to delineated contour and physical examination considering length of treatment, cylinder diameter, and prescribed dose. Both 2D and 3D techniques were used for treatment design. The physics master was blinded to 2D plan when performing 3D design in relation to contour and optimized dose based on the best coverage of CTV and OARs' constraints. Moreover, 2D planning was performed to deliver the prescribed dose to a $0.5 \mathrm{~cm}$ depth of the vagina, and optimized when $150 \%$ isodose was located at the cylinder surface and $200 \%$ inside the cylinder. This type of planning was performed without any imaging after which, a defined dwell time on 2D planning was performed on CT images contoured as previously described. Dwell points with a $3.5 \mathrm{~mm}$ distance on 2D plans were maintained in all cases, while their data were transferred to $\mathrm{CT}$ images. Therefore, 2D plan was copied on CT images, and dose constraints were consequently measurable. The patients received 4 to $7 \mathrm{~Gy}$ in 3 to 5 fractions, with one-week interval, using the same treatment plan designated for the first fraction. HDR Plus v. 8.2.3 ${ }^{\circledR}$ software, TG-43 algorithm for dose calculation, and ${ }^{60} \mathrm{Co}$ source (MultiSource, Eckert-Ziegler ${ }^{\circledR}$, Belgium) were used for brachytherapy.

\section{Statistical analysis}

$D_{90}$ and $D_{99}$ (defined as the dose delivered to $90 \%$ and $99 \%$ of the target volume, respectively) as well as $V_{100}$, $\mathrm{V}_{150}$, and $\mathrm{V}_{200}$ (defined as the volume of target, which received $100 \%, 150 \%$, and $200 \%$ of the prescribed dose) were considered. $D_{0.1 c c^{\prime}} D_{1 c c^{\prime}}$ and $D_{2 c c}$ (defined as the dose received by $0.1,1$, and 2 cc of an OAR) were also calculated to evaluate OARs dosimetry. Data were recorded in SPSS software version 16 and compared between 2D and 3D plans using Kolmogorov-Smirnov test to check normality. Moreover, a parametric test (paired sample $t$-test) and non-parametric test (Wilcoxon ranks test) were applied to compare variables between 3D and 2D plans. Mean, median, standard deviation, and interquartile range were calculated for all quantitative data, and frequency and percentage of qualitative data were assessed. $P$-values under 0.05 were considered significant.

\section{Results}

\section{Population}

In total, 41 patients with stage I-II endometrial cancer were enrolled; however, three cases were excluded due to findings of cervix residue on CT scans, and another patient was excluded due to a failure in attending CT scan. Finally, 37 patients were enrolled in the present study, with $15(40.5 \%)$ stage I and 22 (59.5\%) stage II cases. The mean patients' age was $62 \pm 3.5$ years. Twenty-six patients $(70.3 \%)$ were grade $1 / 2$, and 11 patients $(29.7 \%)$ were grade 3 . Both $2 \mathrm{D}$ and 3D plans were designed with equal dose according to a physician's order, and the mean and median vaginal length, target volume, and prescribed dose in each fraction were $51.09 \pm 2.3 \mathrm{~mm}$, $49.1 \pm 2.1 \mathrm{~mm}, 23.83 \pm 1.9 \mathrm{cc}, 21.7 \mathrm{cc}$, and $5.95 \pm 0.11 \mathrm{~Gy}$, and $6 \mathrm{~Gy}$, respectively.

\section{Comparing parameters in $2 D$ vs. $3 D$ plans}

Figure 1 shows the target volume, OARs, and isodose lines in 2D and 3D plans in one patient. First, parameters of the target volume dosimetry were analyzed. Our results showed that the mean amount of both $\mathrm{D}_{90}$ and $\mathrm{D}_{99}$ were significantly higher in 2D plans comparing with 3D plans. Furthermore, although $\mathrm{V}_{100}$ showed no significant difference between $2 \mathrm{D}$ and $3 \mathrm{D}$ plans $(p>0.05), \mathrm{V}_{150}$ and $\mathrm{V}_{200}$ were considerably higher in $2 \mathrm{D}$ plans compared to 3D plans. Table 1 shows a comparison of $\mathrm{D}_{90}, \mathrm{D}_{99}, \mathrm{~V}_{100}$, $\mathrm{V}_{150}$, and $\mathrm{V}_{200}$ between $2 \mathrm{D}$ and $3 \mathrm{D}$ plans.

Second, parameters suggesting the delivered dose to OARs, including $\mathrm{D}_{0.1 \mathrm{cc}}, \mathrm{D}_{1 \mathrm{cc}}$ and $\mathrm{D}_{2 \mathrm{cc}}$ were compared through a non-parametric test between the two groups of patients. As shown in Table 2, all parameters regarding dose delivered to the rectum and sigmoid were lesser in $3 \mathrm{D}$ than $2 \mathrm{D}$ plans ( $p$-value $<0.05)$. The dose delivered to the bladder analyzed by the parametric test was lesser in $3 \mathrm{D}$ plans; however, $\mathrm{D}_{0.1 \mathrm{cc}}$ was not statistically significant ( $p$-value $>0.05)$, which means that $3 \mathrm{D}$ planning could cause the rectum sigmoid and bladder to receive a reduced amount of dose.

\section{Discussion}

High-dose-rate BRT plays a significant role in adjuvant treatment of early-stage endometrial cancer [8, 9]. Although radiation therapy reduces the risk of vaginal cuff relapse, it does not impact overall survival [8]. PORTEC-2 trial was conducted to find practical and less toxic treatment method, and reported that HDR-BRT could be used as the standard of treatment, since it can reduce the risk of local relapse as well as EBRT [8]. HDRBRT could be performed through a BRT cylinder, single-channel, or multi-channel cylinder. A single-channel cylinder is the most common way to cover the vagina. However, a multi-channel cylinder can spare normal tissue more than a single channel due to its' feasibility of deactivating sources near OARs [11, 23, 24]. As early-stage endometrial cancer patients treated with single-channel HDR-BRT would experience a prolonged survival, administration of a less toxic treatment method is essential. 
2D plan



3D plan

\$2 $<3>Y Z$

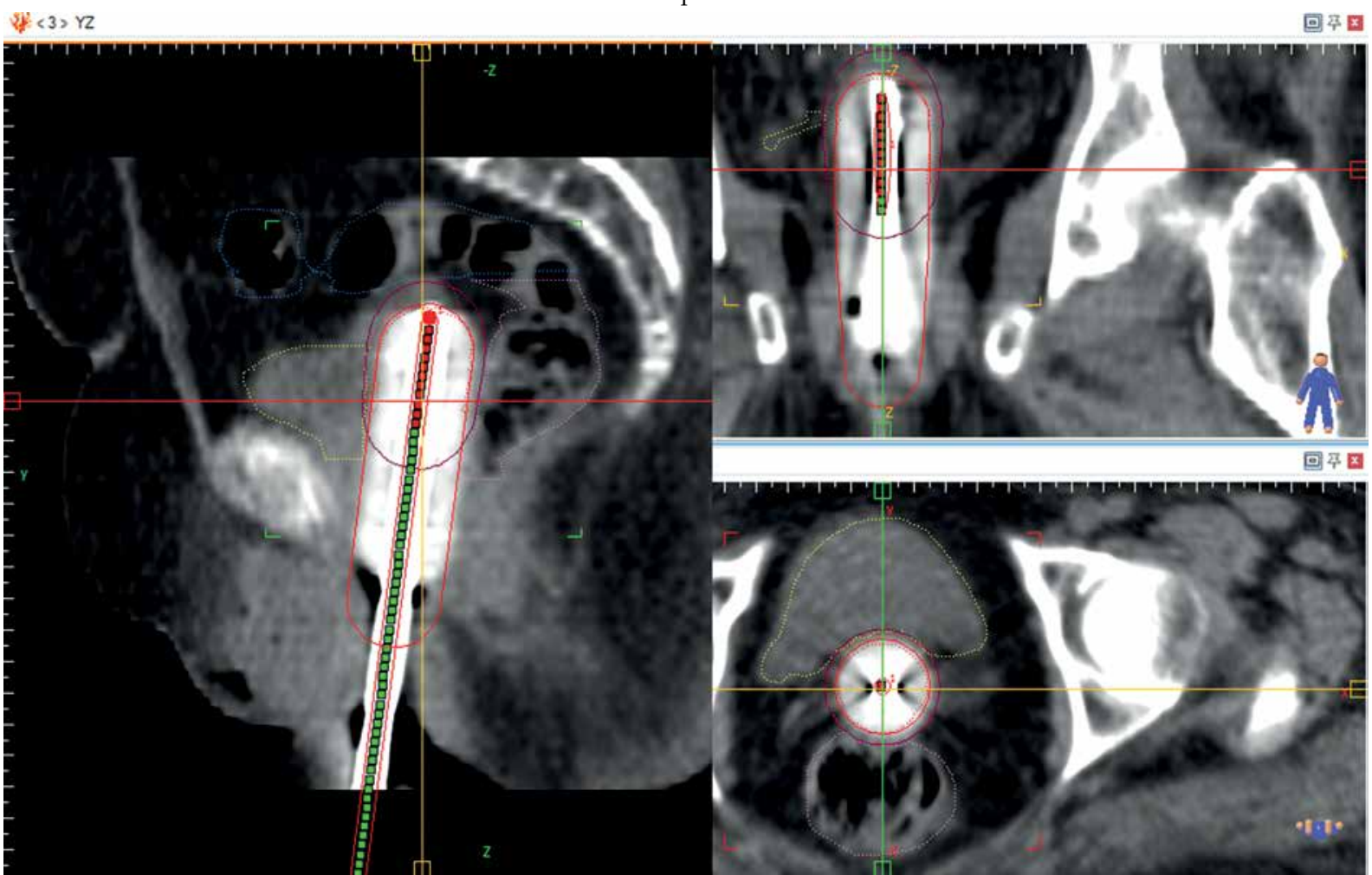

Fig. 1. Target volume, organs-at-risk (OARs), and isodose lines in 2D and 3D plans. The bladder, rectum, and sigmoid are contoured with yellow, pink, and blue lines, respectively. Activated sources and target volume are shown with red color. Isodose 100 is purple, which is tangential to the target volume in 3D; however, this line is beyond the target volume in 2D. In addition, as it is shown in the pictures, this line passed a lesser volume of OARs in 3D plans 
Table 1. Comparison of $\mathrm{D}_{90}, \mathrm{D}_{99}, \mathrm{~V}_{100}, \mathrm{~V}_{150}$, and $\mathrm{V}_{200}$ between $2 \mathrm{D}$ and $3 \mathrm{D}$ planes

\begin{tabular}{lccc} 
CTV parameters in HDR-BRT & 2D $($ mean \pm SD $)$ & 3D $($ mean \pm SD $)$ & $p$-value \\
\hline $\mathrm{D}_{90}$ & $5.9 \pm 1.2 \mathrm{~Gy}$ & $5.6 \pm 1.2 \mathrm{~Gy}$ & 0.04 \\
\hline $\mathrm{D}_{99}$ & $5.0 \pm 1.3 \mathrm{~Gy}$ & $4.7 \pm 1.3 \mathrm{~Gy}$ & 0.03 \\
\hline $\mathrm{V}_{100}$ & $0.88 \pm 0.11$ & $0.84 \pm 0.14$ & 0.06 \\
\hline $\mathrm{V}_{150}$ & $0.25 \pm 0.13$ & $0.19 \pm 0.13$ & 0.02 \\
\hline $\mathrm{V}_{200}$ & $0.06 \pm 0.05$ & $0.04 \pm 0.03$ & $<0.001$
\end{tabular}

Table 2. Comparing $D_{\max }, D_{0.1 c c}, D_{1 c c}$, and $D_{2 c c}$ in rectum, sigmoid, and bladder in $2 D$ and $3 D$ planes

\begin{tabular}{lccc} 
Organ & 2D (mean $\pm S D)(G y)$ & $3 D($ mean $\pm S D)(G y)$ & $p$-value \\
\hline Rectum & & & 0.01 \\
\hline$D_{0.1 c c}$ & $7.28 \pm 2.28$ & $6.72 \pm 2.11$ & $<0.001$ \\
\hline$D_{1 c c}$ & $5.85 \pm 1.30$ & $5.32 \pm 1.30$ & $<0.001$ \\
\hline$D_{2 c c}$ & $5.40 \pm 1.10$ & $4.77 \pm 1.12$ & $<0.001$ \\
\hline Sigmoid & & & $<0.001$ \\
\hline$D_{0.1 c c}$ & $6.40 \pm 2.50$ & $5.30 \pm 2.04$ & $<0.001$ \\
\hline$D_{1 c c}$ & $4.66 \pm 1.70$ & $4.06 \pm 1.50$ & 0.17 \\
\hline$D_{2 c c}$ & $4.16 \pm 1.50$ & $3.61 \pm 1.35$ & 0.04 \\
\hline Bladder & & & $5.80 \pm 1.50$ \\
\hline$D_{0.1 c c}$ & $6.14 \pm 1.60$ & $4.75 \pm 1.30$ & 0.05 \\
\hline$D_{1 c c}$ & $5.00 \pm 1.30$ & $4.34 \pm 1.9$ & \\
\hline$D_{2 c c}$ & $4.55 \pm 1.2$ & & \\
\hline
\end{tabular}

Using CT scan imaging in 3D planning to reduce toxicity of HDR-BRT was evaluated in several studies. However in the present study, it was the first time that the thickness of target volume was contoured based on CT scan slices, and the length of vagina was modulated based on clinico-pathological aspects.

Globally, two-dimensional planning is the most common method of treatment. The reason for not using cross-sectional images in 2D plans was that dose gradient falls sharply around brachytherapy cylinder's sources. Here, we challenged this idea to determine whether using CT scan images can improve dose delivered to target volume and reduce dose to OARs. This hypothesis should be examined by comparing dose received through $3 \mathrm{D}$ planning, which optimizes dose on contouring and plan designation, according to activity sources in 2D plan on CT scan images. As discussed in previous studies, there are different optimization methods in 2D plan, including normalization point in the lateral aspect of vagina, ICRU point, setting normalization point at the surface of cylinder, or at a $5 \mathrm{~mm}$ depth of vagina. In the present study, we applied ${ }^{60} \mathrm{Co}$ in the treatment of our patients; optimized 2D plans were based on $5 \mathrm{~mm}$ depth of vagina and dosimetry parameters of 2D plan and 3D plan of each patient were compared.

A comparison of $\mathrm{D}_{90}$ and $\mathrm{D}_{99}$ between $2 \mathrm{D}$ and $3 \mathrm{D}$ plans has shown that although mean $D_{90}$ and $D_{99}$ were more in $2 \mathrm{D}$ than $3 \mathrm{D}$ plans, only six patients received $\mathrm{D}_{90}$ less than $90 \%$ in 3D plans compared to nine patients in the 2D group. Similar findings were reported by Kim et al. [13] who reported mean percentage of $\mathrm{D}_{90}$ at around $106 \%$ in $2 \mathrm{D}$ comparing with $103 \%$ in $3 \mathrm{D}$ plans. The same results were shown by a study by Gultekin et al. [16] who stated that $\mathrm{D}_{90}, \mathrm{D}_{95}$, and $\mathrm{D}_{100}$ were less in $3 \mathrm{D}$ plans compared to 2D plans, when normalization point was chosen in a $5 \mathrm{~mm}$ depth of the vagina. Moreover, Zhang et al. [25] showed that tumor cell distribution and shape of the target should be considered in radiation therapy designation due to radio-biologic effects. Based on our work and analyzing $\mathrm{V}_{100}, \mathrm{~V}_{150}$, and $\mathrm{V}_{200}$, it was shown that $\mathrm{V}_{100}$ was approximately equal between 2D and 3D plans, even though $V_{150}$ and $V_{200}$ were less in 3D plans. This means that $3 \mathrm{D}$ plans not only could deliver the prescribed dose to more volume of the target volume, but also was able to spare more volume of the vagina from receiving an excess dose. Kim et al. [13] demonstrated that both $\mathrm{V}_{100}$ and $\mathrm{V}_{150}$ were less in $3 \mathrm{D}$ plans in comparison with $2 \mathrm{D}$ plans.

Another advantage of using CT scan imaging and 3D planning is that, since there were no organs' sectioning in 2D plans, therefore, calculation and judgment of the delivered dose to target and OARs were impossible. Russo et al. [15] used ICRU point dose for bladder and rectum and could not accurately estimate dose delivered to OARs. They concluded that dose calculated through ICRU point was less than $\mathrm{D}_{2 \mathrm{cc}}$ and $\mathrm{D}_{\text {max }}$ in bladder and rectum. Kim et al. [13] and Gultekin et al. [16] also revealed that dose delivered to these organs, including $\mathrm{D}_{0.1 \mathrm{cc}}, \mathrm{D}_{1 \mathrm{cc}}$, and $\mathrm{D}_{2 \mathrm{cc}}$ were smaller in 3D plan rather than 2D plan.

Computed tomography (CT) scan imaging can yield information about the position and status of vaginal vault. In the present study, 3 out of 41 patients were diagnosed with residual cervix during CT scan imaging. They were excluded due to impossibility of covering the remained cervix through brachytherapy cylinder as well as the 
position of cylinder and potential air gaps between the cylinder and vaginal cuff. In a study by Humphrey et al. [14], CT scan imaging after cylinder placement could exhibit air gaps, which might be modified in a next imaging setup. They showed that CT scans could be used as a guide to choose the most fitting cylinder to occupy vaginal space. Similar findings were reported by Sikorska et al., where it was shown that CT scan could help choosing the best cylinder size, resulting in air gaps decreasing [26]. Another study with CT scan performed by Marcos et al. [27] at the Johns Hopkins University revealed that approximately half of patients need cylinder repositioning between HDR-BRT fractions, suggesting that CT scan imaging can guide physicians to place the applicator in the best position.

Although CT scan imaging-based planning has several advantages over 2D planning, it has some limitations. First, CT scanning is a time-consuming and expensive procedure, and may require additional workload in brachytherapy departments. Second, its power to discriminate soft tissues is lesser than MRI. Finally, the possibility of cylinder repositioning between fractions makes it necessary to repeat CT scanning in each fraction.

Since early-stage endometrial cancer is highly curable, particular attention should be paid to reduce treatment toxicities. This study demonstrated that 3D planning can deliver a suitable dose to the target while sparing OARs. Furthermore, it was the first time that ${ }^{60} \mathrm{Co}$ was applied in a comparison of $2 \mathrm{D}$ and $3 \mathrm{D}$ planning, showing that $3 \mathrm{D}$ planning could be beneficial in developing countries.

\section{Conclusions}

The present study indicate that using CT scan imaging to perform brachytherapy planning can deliver suitable dose to the target volume while sparing OARs, especially rectum and sigmoid. In addition, imaging before dose delivery to target volume can reveal the position of cylinder in the vagina, and provide an information whether the cylinder is capable of covering the target.

\section{Disclosure}

The authors report no conflict of interest.

\section{References}

1. World Health Organization. Corpus uteri fact sheet: International agency for research on cancer; 2020. Available from: https://gco.iarc.fr/today/data/factsheets/cancers/24-Corpus-uteri-fact-sheet.pdf.

2. Society AC. Key statistics for endometrial cancer USA: American Cancer Society 2020. Available from: https:/ / www.cancer.org/cancer/endometrial-cancer/about/key-statistics.

3. Treatment NCIEC. Endometrial Cancer Treatment. Physician Data Query (PDQ) 2015. Available from: http://www. cancer. gov/cancertopics/pdq/treatment/endometrial/ healthprofessional.

4. Creutzberg CL, van Putten WL, Koper PC et al. Surgery and postoperative radiotherapy versus surgery alone for patients with stage-1 endometrial carcinoma: multicentre randomised trial. PORTEC Study Group. Post Operative Radiation Therapy in Endometrial Carcinoma. Lancet 2000; 355: 1404-1411.
5. Keys HM, Roberts JA, Brunetto VL et al. A phase III trial of surgery with or without adjunctive external pelvic radiation therapy in intermediate risk endometrial adenocarcinoma: a Gynecologic Oncology Group study. Gynecol Oncol 2004; 92: 744-751.

6. Canlorbe G, Bendifallah S, Laas E et al. Tumor size, an additional prognostic factor to include in low-risk endometrial cancer: results of a French multicenter study. Ann Surg Oncol 2016; 23: 171-177.

7. Nout RA, van de Poll-Franse LV, Lybeert M et al. Long-term outcome and quality of life of patients with endometrial carcinoma treated with or without pelvic radiotherapy in the post operative radiation therapy in endometrial carcinoma 1 (PORTEC-1) trial. J Clin Oncol 2011; 29: 1692-1700.

8. Nout RA, Smit V, Putter H et al. Vaginal brachytherapy versus pelvic external beam radiotherapy for patients with endometrial cancer of high-intermediate risk (PORTEC-2): an open-label, non-inferiority, randomised trial. Lancet 2010; 375: 816-823.

9. Wortman BG, Creutzberg CL, Putter $\mathrm{H}$ et al. Ten-year results of the PORTEC-2 trial for high-intermediate risk endometrial carcinoma: improving patient selection for adjuvant therapy. Br J Cancer 2018; 119: 1067-1074.

10. Foerster R, Schnetzke L, Bruckner T et al. Prognostic factors for long-term quality of life after adjuvant radiotherapy in women with endometrial cancer. Strahlenther Onkol 2016; 192: 895-904.

11. Harkenrider MM, Grover S, Erickson BA et al. Vaginal brachytherapy for postoperative endometrial cancer: 2014 Survey of the American Brachytherapy Society. Brachytherapy 2016; 15: 23-29.

12. DeVita VT, Lawrence TS, Rosenberg SA. DeVita, Hellman, and Rosenberg's cancer: principles and practice of oncology. 11 ed. Wolters Kluwer Health 2015.

13. $\mathrm{Kim} \mathrm{H}, \mathrm{Kim} \mathrm{H}$, Houser $\mathrm{C}$ et al. Is there any advantage to three-dimensional planning for vaginal cuff brachytherapy? Brachytherapy 2012; 11: 398-401.

14. Humphrey P, Cornes P, Al-Booz H. Vaginal vault brachytherapy in endometrial cancer: verifying target coverage with image-guided applicator placement. Br J Radiol 2013; 86: 20120428.

15. Russo JK, Armeson KE, Richardson S. Comparison of 2D and $3 \mathrm{D}$ imaging and treatment planning for postoperative vaginal apex high-dose rate brachytherapy for endometrial cancer. Int J Radiat Oncol Biol Phys 2012; 83: e75-e80.

16. Gultekin M, Yilmaz MT, Biltekin F et al. Adjuvant vaginal cuff brachytherapy: dosimetric comparison of conventional versus 3-dimensional planning in endometrial cancer. J Contemp Brachytherapy 2020; 12: 601-605.

17. Strohmaier S, Zwierzchowski G. Comparison of (60)Co and (192)Ir sources in HDR brachytherapy. J Contemp Brachytherapy 2011; 3: 199-208.

18. Tantivatana T, Rongsriyam K. Treatment outcomes of highdose-rate intracavitary brachytherapy for cervical cancer: a comparison of Ir-192 versus Co-60 sources. J Gynecol Oncol 2018; 29: e86.

19. Mosalaei A, Mohammadianpanah M, Omidvari S et al. Highdose rate brachytherapy in the treatment of carcinoma of uterine cervix: twenty-year experience with cobalt after-loading system. Int J Gynecol Cancer 2006; 16: 1101-1105.

20. Ntekim A, Adenipekun A, Akinlade B et al. High dose rate brachytherapy in the treatment of cervical cancer: preliminary experience with cobalt 60 radionuclide source - a prospective study. Clin Med Insights Oncol 2010; 4: 89-94.

21. Bocharova I. The history of brachytherapy in Russia: comparison of Co-60 vs. Ir-192 sources. J Contemp Brachytherapy 2011; 3: 48-49. 
22. Sealed EL. Power calculator for binary outcome non-inferiority trial. https://www.sealedenvelope.com/power/binary-noninferior/ 2012.

23. Tanderup K, Lindegaard JC. Multi-channel intracavitary vaginal brachytherapy using three-dimensional optimization of source geometry. Radiother Oncol 2004; 70: 81-85.

24. Bahadur YA, Constantinescu C, Hassouna AH et al. Single versus multichannel applicator in high-dose-rate vaginal brachytherapy optimized by inverse treatment planning. J Contemp Brachytherapy 2015; 6: 362-370.

25. Zhang H, Donnelly ED, Strauss JB et al. Therapeutic analysis of high-dose-rate 192Ir vaginal cuff brachytherapy for endometrial cancer using a cylindrical target volume model and varied cancer cell distributions. J Med Phys 2016; 43: 483-494.

26. Sikorska K, Zolciak-Siwinska A, Kowalczyk A et al. Dosimetric evaluation of vaginal cuff brachytherapy planning in cervical and endometrial cancer patients. J Contemp Brachytherapy 2020; 12: 248-251.

27. Morcos M, Moore J, Rezaee M et al. Efficient vaginal cylinder brachytherapy: forgoing daily re-planning with mobile CT image-guidance. Int J Radiat Oncol Biol Phys 2019; 105: E733. 